Europhysics Letters

PREPRINT

\title{
On two intrinsic length scales in polymer physics: topological constraints vs. entanglement length
}

\author{
M. MÜller ${ }^{1}$, J.P. Wittmer ${ }^{2}\left(^{*}\right)$ and J.-L. Barrat ${ }^{2}$ \\ 1 Institut für Physik, Johannes Gutenberg-Universität, Staudinger Weg 7, D-55099 \\ Mainz, Germany. \\ 2 Département de Physique des Matériaux, Université Claude Bernard and CNRS, \\ 69622 Villeurbanne Cedex, France.
}

PACS. $61.25 . \mathrm{Hq}$ - Macromolecular and polymer solutions.

PACS. 83.20.Fk - Reptation theories.

PACS. 83.10.Nn - Polymer dynamics.

\begin{abstract}
The interplay of topological constraints, excluded volume interactions, persistence length and dynamical entanglement length in solutions and melts of linear chains and ring polymers is investigated by means of kinetic Monte Carlo simulations of a three dimensional lattice model. In unknotted and unconcatenated rings, topological constraints manifest themselves in the static properties above a typical length scale $d_{t} \sim 1 / \sqrt{l \phi}$ ( $\phi$ being the volume fraction, $l$ the mean bond length).

Although one might expect that the same topological length will play a role in the dynamics of entangled polymers, we show that this is not the case. Instead, a different intrinsic length $d_{e}$, which scales like excluded volume blob size $\xi$, governs the scaling of the dynamical properties of both linear chains and rings. In contrast to $d_{t}, d_{e}$ has a strong dependence on the chain stiffness. The latter property enables us to study the full crossover scaling in dynamical properties, up to strongly entangled polymers. In agreement with experiment the scaling functions of both architectures are found to be very similar.
\end{abstract}

Introduction. Topological constraints (TC) are commonly invoked in polymer theory to explain the dynamical behaviour of large molecular weight chains. In the reptation theory [1] - 4 , the long range TC are assumed to be responsible on a local scale for the conjectured anisotropic motion of chain segments, which are not allowed to cross neighboring chains. Obviously, in systems of linear chains this non-crossing constraint does not provide TC in a strict mathematical sense [2]. Strict topological constraints, however, do exist in systems of unconcatenated and unknotted ring polymers, where they strongly influence the static properties [5]. In this case, the $\mathrm{TC}$ are created by the non-crossing requirement and, as has been shown recently [8], a mass independent length scale $d_{t}$. It is, hence, natural to ask whether $d_{t}$ provides the conjectured length scale, i.e. the "tube diameter" $d_{e}$ [2], for the dynamics of linear chains and rings. A link between the two concepts was indeed suggested by the fathers of the reptation concept, de Gennes [1] and Edwards [2]. This idea prompted the

$\left({ }^{*}\right)$ E-mail: jwittmer@dpm.univ-lyon1.fr

(c) EDP Sciences 


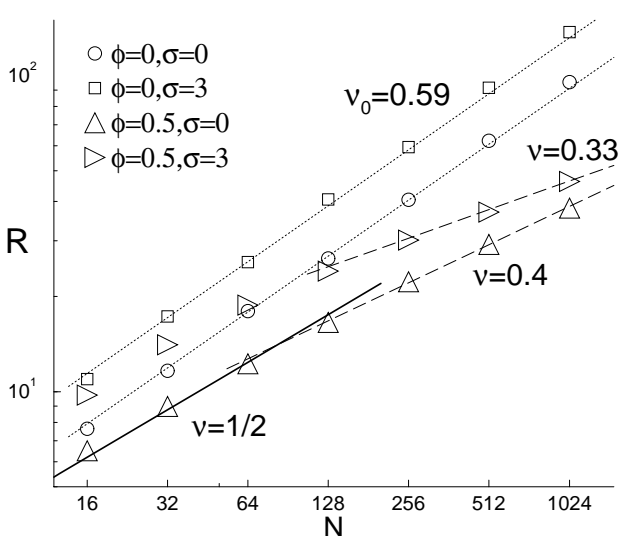

Fig. 1

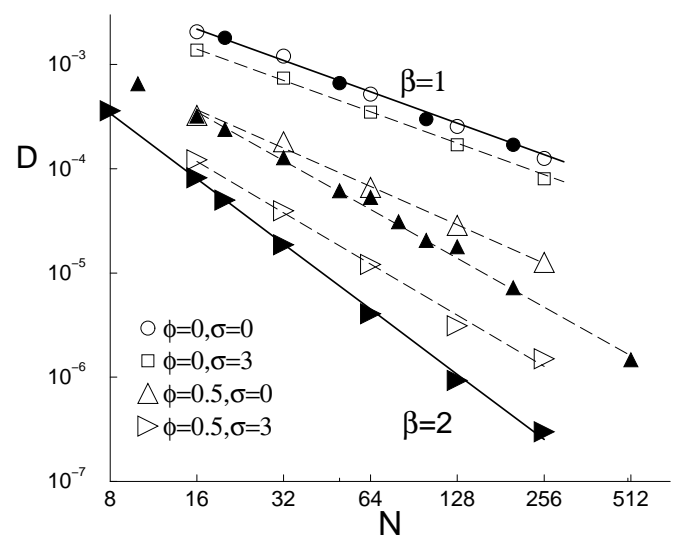

Fig. 2

Fig. $1-$ Ring size $R=\left\langle\vec{R}_{0}-\vec{R}_{0+N / 2}\right\rangle^{1 / 2}$ versus chain length $N$ for dilute $(\phi \rightarrow 0)$ and molten rings. The dilute rings are characterized by the same Flory exponent $\nu=\nu_{0} \approx 0.59$ as their linear counterparts (not included). In the dense limit short rings form Gaussian chains of blobs $(\nu=1 / 2$ : solid line). With chain overlap, i.e. with increasing molecular mass, volume fraction and (most importantly) stiffness, the rings become more and more compact.

Fig. 2 - Diffusion coefficient $D(N, \phi, \sigma)$ versus chain length $N$. We consider flexible $(\sigma=0)$ and semiflexible $(\sigma=3)$ linear chains (full symbols) and rings (empty symbols) in the dilute and the melt limit. In the dilute limit both architectures have the same Rouse-like dynamics (upper bold line). Contrasting to this the dynamics at high overlap depends strongly on both architecture and stiffness. Effective power law exponents $D \propto N^{-\beta}$ are indicated in the figure (dashed lines); $\beta$ increases with $N, \phi$ and $\sigma$. Rings are always faster than corresponding linear chains and have smaller apparent exponents. The $\beta \approx 2$-slope - consistent with the reptation prediction [1, 2] - is observed over more than one order of magnitude for semiflexible $(\sigma=3)$ linear chains (lower bold line).

present computational study, which extends our previous investigation on semiflexible rings on linear polymers. Concentrating on the scaling analysis of global quantities such as the diffusion constant and the radius of gyration, we demonstrate that the static and dynamic problems involve different length scales, with different dependence on chain stiffness and on density. The conjecture $d_{e} \approx d_{t}$ is therefore incorrect, and the simpler proposal $d_{e} \approx \xi$ [2, 化, where $\xi$ is the correlation blob size, accounts much better for the scaling behaviour.

Computational method and raw data. As in our previous studies [8, 9], we use Monte-Carlo simulations of an extensively studied lattice model, the bond fluctuation model [10]. This model, in which the dynamics proceeds by local jump attempts, is thought to describe well the polymer dynamics on Langevin equation level [11]. In order to tune the persistence length, we impose a simple intramolecular potential which favors straight bond angles $E(\theta)=\sigma \cos (\theta)$ where $\theta$ denotes the complementary angle between two successive bonds. The stiffness $\sigma$ (in the range $\sigma=0,1,2$ and 3 ) is an energy scale, expressed here in units of the thermal energy $k_{B} T$, 8, 9 .

In fig. 1 and fig. 2 we present two sets of raw data from our simulations - the ring size $R$ and the self-diffusion constant $D$ for linear chains and rings as functions of their molecular mass $N$. We have sampled a broad range of volume fractions (of occupied lattice sites) $\phi$ from the dilute up to the melt [11] limit $(\phi \approx 1 / 2)$ with chain masses up to $N=1024$ in order to vary the chain overlap sufficiently to test the proposed scaling scenarios. 
In the bond fluctuation model the bond length $l$ is not, strictly speaking, a constant, since a bond can be represented by lattice vectors of different lengths. The average bond length $l$, however, depends very weakly on stiffness and density, and will be considered as constant. The data for dilute chains can be used to define the effective or statistical segment length $b_{0}(\sigma)$, obtained from fitting the radius of gyration of asymptotically long dilute linear chains with $R_{g y r}=b_{0}(\sigma) N^{\nu_{0}}$ where $\nu_{0} \approx 3 / 5$ is the swollen chain exponent. $b_{0}(\sigma)$ increases with stiffness up to $50 \%$. Similarly, linear chains above the overlap concentration are Gaussian, and obey the scaling $R_{g y r}=b(\phi, \sigma) N^{1 / 2}$. Local dynamics is characterized by a monomer mobility $m(\phi, \sigma)$, obtained from the mean-square displacements (MSD) of center monomers at short times 9]. Again, this quantity turns out to be relatively insensitive to stiffness. In the dilute limit it may be equally be obtained from the center-of-mass motion. As shown in fig. 2 we find $D=m_{0}(\sigma) / N$ for both architectures, with a mobility $m_{0}$ roughly proportional to the acceptance rate. At higher densities we find $m(\phi, \sigma) \approx m_{0}(\sigma) \tilde{m}(\phi)$ with $\tilde{m}(0)=1$, i.e. density and rigidity effects decouple.

While static and dynamic properties of both architectures resemble each other in the dilute limit and at moderate overlap, where both linear chains and rings form Gaussian chains of blobs - bold line in fig. 1 - marked differences appear at high chain overlap, as seen in figures 1 and 2. The local slopes in these figures, $\nu=d \log R / d \log N$ and $\beta=-d \log D / d \log N$ depend continuously on $N$, indicating that our data extensively cover the crossover regions. With increased chain overlap the rings become more compact $(1 / \nu \rightarrow 3)$ and their dynamics more entangled-like, i.e. the diffusion exponent $\beta$ approaches 2 12]. The stiffness dependence is very strong, especially for the diffusion constant. That the reptation prediction $\beta=2$ for linear chains [1,2] is indeed seen so clearly, is one of the important results of this work; this is in agreement with a very recent MD simulation [13. Note that rings are always faster than their linear counterparts.

Obviously, the slopes are only effective exponents which characterize the crossover between different regimes and do not capture asymptotic behaviour (e.g., the two slopes indicated in fig. 11 would then intercept which is unphysical). The task is then to understand the effects on both architectures of increased chain overlap - specifically, the strong stiffness dependence — and to elucidate the underlying crossover scaling.

Two intrinsic length scales and scaling attempts. There are two [15] natural intrinsic length scales — both chain length independent — which have been used to characterize the interactions in strongly overlapping polymer solutions and melts [1]: the 'diameter' $\rho(\phi)=$ $1 / \sqrt{l \phi}$ and the 'blob' size $\xi\left(\phi, b_{0}\right) \approx b_{0} N_{\xi} \nu_{0}$ where $N_{\xi} \approx\left(b_{0}^{3} \phi\right)^{-1 /\left(3 \nu_{0}-1\right)}$ is the number of monomers contained in the blob. Note that in contrast to $\rho$ the blob size $\xi \propto b_{0}{ }^{-1.3}$ is strongly stiffness dependent. The prefactors of $\xi$ and $N_{\xi}$ can be estimated independently from the static structure factor or, equivalently, from $b(\phi, \sigma)=\xi / N_{\xi}{ }^{1 / 2}$ [8, 9].

We demonstrate now that $\rho$ determines $d_{t}$ (Fig. 3) and that the polymer dynamics is indeed characterized by one length scale $d_{e} \sim \xi$ (Fig. 4 ). Note that the figures include data from very different densities and rigidities and that no shift or fit parameter has been used. It is also important to remark that data points may be found in the different parts of the scaling functions, irrespective of their density or stiffness. This shows that the true intrinsic parameter is neither $\phi$ nor $\sigma$, but the proposed scaling variable.

First, we have verified that the traditional scaling $R / \xi$ versus $N / N_{\xi}$ [1] works for semiflexible linear chains (not shown). Surprisingly, it does not work for rings [8]. This is an indication of the influence of TC on static properties. In fig. 3, we show that a proper scaling of the radius of gyration for rings is obtained by using the scaling variables $R / d_{t}$ and $N / N_{t}$, where $d_{t} \approx 25 / \sqrt{l \phi}$. The number $N_{t}$ of monomers between topological obstacles $N_{t}$ used in 


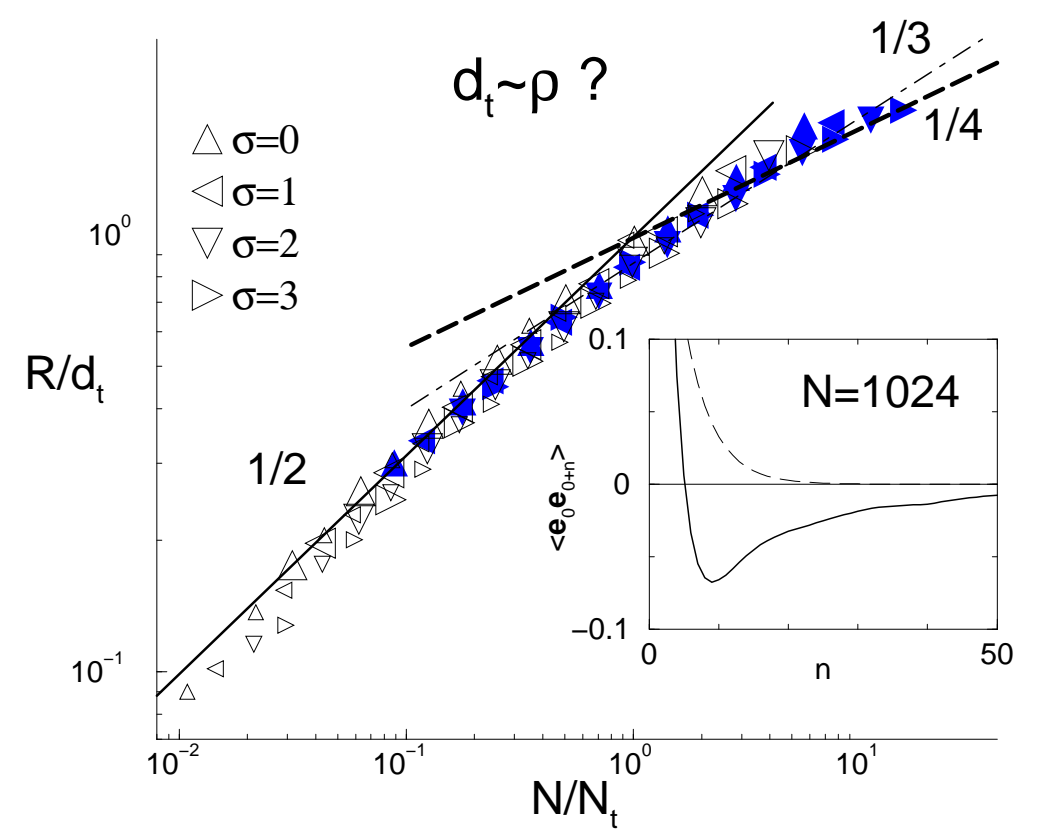

Fig. 3 - Data collapse of the reduced ring size $R / d_{t}$ versus $N / N_{t}$ for rings at three representative densities $(\phi=1 / 2$ : full symbols; $\phi=1 / 8$ : large, thin symbols; $\phi=1 / 32$ : small symbols) at different $\sigma$ as indicated in the figure. The successful scaling attempt supposes $d_{t} \approx 25 \rho$ and self-consistently (full line on the left) Gaussian chains of blobs at small distances, i.e. $N_{t} \approx 405(\rho / b(\phi, \sigma))^{2}$. The slope $\nu=1 / 4$ for the predicted LA asymptotic behaviour for larger chains is indicated on the right (dashed) along with the apparent $\nu=1 / 3$-slope (dashed-dotted) from fig. 11 which fits a broader range of data. Inset: The bond-bond correlation function $\left\langle\vec{e}_{0} \cdot \vec{e}_{0+n}\right\rangle$ shows striking anti-correlations for rings (bold line) at large overlap $(N=1024, \phi=0.5, \sigma=3)$ in contrast to linear chains (dashed).

fig. 3 is obtained from $d_{t} \approx b(\phi, \sigma) N_{t}{ }^{1 / 2}$ where we assume Gaussian statistics at distances smaller $d_{t}$. This is motivated by fig. 1 and is consistent with the scaling (left side of fig. 33). From this successful scaling collapse we may conclude that the length generated by the TC is $d_{t} \approx 25 / \sqrt{l \phi}$. This yields $d_{t} \approx 22$ for $\phi=0.5$ and $N_{t}(\sigma=0) \approx 173$ and $N_{t}(\sigma=3) \approx 63$. This length is both mass and stiffness independent. The prefactor is somewhat arbitrary and depends on the asymptotic slope on the right which is numerically unclear (see below).

We turn now to the scaling of the diffusion data, and demonstrate, that polymer dynamics is indeed characterized by one length scale $d_{e}$ which scales like the blob size $\xi$. In fig. 4 , the mass has been rescaled by a number $N_{e}$ assumed to be proportional to the number of monomers per blob $N_{\xi}$. The scale for the vertical axis is given by $D_{e}=m(\phi, \sigma) / N_{e}$, i.e. we assume Rouse-like behaviour for weakly overlapping chains. For small $N$ all data collapse irrespective of architecture and stiffness on the asymptotic slope with $\beta=1$. This shows that the definition of $D_{e}$ is self-consistent and also that the mobility correction $m(\phi, \sigma)$ was chosen appropriately. For $N \gg N_{e}$ we find that the linear chain data follow the reptation prediction $D / D_{e}=\left(N_{e} / N\right)^{2}$ for nearly two orders in magnitude! From the known blob size and the intercept of both asymptotes one estimates $N_{e} / N_{\xi} \approx 15$ for the linear chains. Note that our scaling is consistent with the traditional density crossover scaling assumption |2] and recent experiments on linear chains 14, 15]. Also, the present scaling, in contrast to that in Ref. [13], 


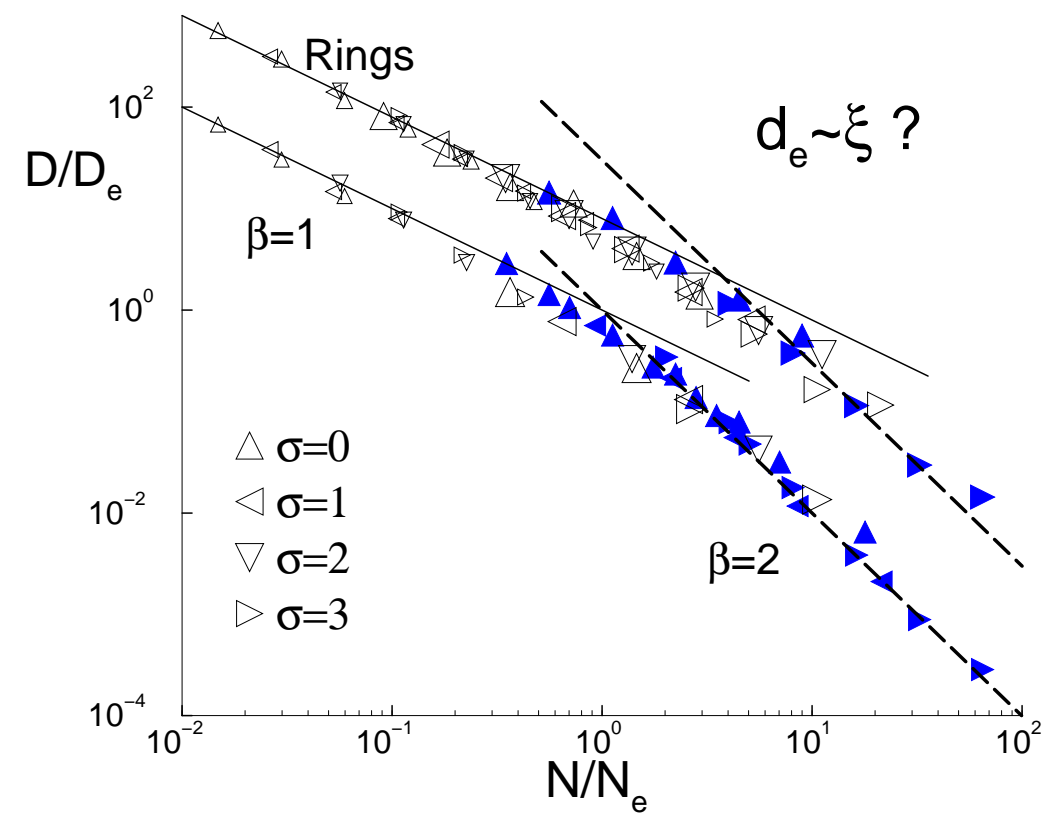

Fig. 4 - Scaling attempt for diffusion constants of linear chains and rings (shifted upwards for clarity) supposing $d_{e} \sim \xi$. $D / D_{e}$ is plotted versus $N / N_{e}$ where we use $D_{e}=m(\phi, \sigma) / N_{e}$ and $N_{e}=32.8\left(b_{0}^{3} \phi\right)^{-1 /\left(3 \nu_{0}-1\right)}$. Data for four rigidities (as indicated in the figure) and three densities (as in fig. 3; same symbols) have been included. The data collapse was achieved without any free parameter! The linear chain data follow the reptation prediction for nearly two orders of magnitude. The prefactor of $N_{e}$ was defined such that both linear chain asymptotes (bold and dashed lines) intercept at $D / D_{e}=N / N_{e}=1$.

does not involve any arbitrary shift parameters.

The universal functions for the dynamical properties of both architectures are similar. In agreement with experiment [3,16] ring dynamics becomes entangled-like at slightly higher chain length. This is no surprise and may be attributed to their smaller size [3]. However, the asymptotic behaviour is less obvious. The best fit for the available data $\beta \approx 1.7$, may be regarded as lower bound. Motivated by recent experiments [16] which show the same asymptotic behaviour for both architectures we have also fitted the ring data with $\beta=2$ yielding a reasonable agreement (upper dashed line in fig. (1). From the intercept of the unentangled and entangled asymptote one concludes that the ring crossover occurs roughly at 3-4 times larger chain length than in their linear counterparts - again in agreement with experiment [16]. This finally explains why the $\beta$ exponents of rings at given $\phi$ and $\sigma$ are smaller.

Interpretation of scalings results and new queries. Both presented scaling plots are consistent with two eminent mean-field descriptions in polymer physics - the lattice animal (LA) proposal for dense rings [5, 6, 8] and the reptation concept for polymer dynamics. They provide additional information in that they identify the phenomenological length scales implicit in both theories.

Our compact rings at high chain overlap behave like LA in a self-consistent network imposed by the TC of neighboring rings, i.e. they may be described on the same footing as isolated rings in a network of fixed topological obstacles of density $1 / d_{t}^{3}$ [6]. If the ring size 
$R$ becomes larger than $d_{t}$ the rings are forced to retrace their paths and the fractal dimension $1 / \nu$ becomes that of a strongly branched object. Within the single chain model it is known that the rings are characterized by the exponent $\nu=1 / 4$ within an intermediate, but broad chain length window [6]. It is this suggestion we have followed in fig. 3 to fix the prefactor of $d_{t}$. Note that the rings presented in fig. 3 are strongly overlapping, with $R$ much larger than the distance between chains (see 8], fig.7), and that we refer to 'compactness' in the scaling sense, $\nu \leq 1 / 3$.

The effective topological interactions can be visualized directly with the bond-bond vector correlation function shown in the inset of fig. 3. This function has a pronounced anticorrelation. The negative minimum indicates that the ring has to fold back after $n \approx 10$ monomers to retrace its path. The position and the depth of the dip increase with $\phi$ and $\sigma$, but are chain length independent which underlines the $N$-independence of $d_{t}$. The $d_{t} \sim \rho$ relation reflects the topological origin of the interaction. Indeed $\rho$ characterizes the volume per chain segment which is invariant with regard to chain conformation, chain length and stiffness. The advantage in using stiff chains is related to the fact that $d_{t}$ does not depend on stiffness: semiflexible rings 'waste' fewer monomers on short length scales, may explore larger distances and become more compact. Tuning the stiffness provides therefore a much more efficient route to increase the chain overlap, required for the mean-field picture to hold, than a chain length variation [8].

Confirming older [9] and more recent simulations [8,13] we find that increasing the stiffness is an even more efficient trick for exploring the strongly entangled dynamics. This is now rationalized by the scaling $d_{e} \sim \xi$ in fig. A which implies that the strong stiffness dependence of the number of entanglements per chain $N / N_{e} \propto b_{0}{ }^{3.9}$ 17]. We emphasize, however, that we have not demonstrated in this study the reptation proposal. The $\beta=2$ asymptote of fig. 1 is not sufficient to claim anisotropic motion of the chains along their contour [3] which requires, e.g., a more detailed analysis of the MSD. This must take into account the non-asymptotic behaviour in the MSD generated by tube renewal [18] and constraint release effects. The latter mechanism is likely to be the dominant relaxation mode for our entangled rings. What has been demonstrated here in a computational study covering, for the first time, several orders of magnitude is (i) the existence of two distinct dynamical regimes, both in linear chains (consistent with [4, 9, 11, 13]) and ring polymers, (ii) that the dynamical crossover is characterized by one length scale only, (iii) that this length scale is the size of the excluded volume blob, i.e. a properly defined static quantity [19, (iv) that, surprisingly, the crossover scaling does not depend on the architecture. However, the finding $d_{t} \gg d_{e}$ for the strongly overlapping chains in our simulations may explain naturally why the length scale $d_{t}$ appears to play no role in the dynamics of rings of experimental [16] and computational relevance.

Summary. In this letter, we have elucidated, by means of a numerical experiment, the scaling of static and dynamic properties of strongly entangled linear chains and rings with regard to density and stiffness variation. Our results indicate that topological constraints manifest themselves differently in the statics and in the dynamics. The topological length which governs the crossover in the statics of rings is essentially independent of stiffness. This contrasts with the strong stiffness dependence of the dynamical entanglement length, which governs the crossover to reptation-like behaviour. Although the high molecular weight diffusion exponent agrees with the reptation prediction, we have not presented rigorous proofs for the anisotropic motion of polymer chains. A very puzzling question in this context is the similarity between the scaling functions for linear chain and ring dynamics which is the focus of forthcoming work. 
We thank M.E. Cates, K. Binder, W. Paul and J. Baschnagel for stimulating discussions. We are grateful to T. Kreer and J. Baschnagel for communication of the diffusion constant $D(N=512, \phi=0.5, \sigma=0)$ prior to publication. Generous grants of CPU time on the CRAY T3E computers at the HLR Stuttgart and the IDRIS Saclay as well as financial support by the ESF under SIMU grant are gratefully acknowledged.

\section{REFERENCES}

[1] De Gennes P.-G., Scaling Concepts in Polymer Physics (Cornell University, Ithaca, N.Y.)1979.

[2] Dor M. and Edwards S. F., The Theory of Polymer Dynamics (Clarendon Press, Oxford) 1986.

[3] Lodge T. P., Rotstein N. A. and Prager S., Advances in Chemical Physics, 79 (1990) 1.

[4] Kremer K. and Grest G., Monte Carlo and Molecular Dynamics Simulations in Polymer Science (Oxford University Press), edited by K. Binder1995; Pütz M. et al.,Europhys. Lett., 49 (2000) 735.

[5] Cates M. E. and Deutsch J. M., J. de Physique, 47 (1986) 2121.

[6] Obukhov S. P., Rubinstein M. and Duke T., Phys.Rev.Lett., 73 (1994) 1263.

[7] Müller M, Wittmer J.P. and Cates M. E., Phys.Rev.E, 53 (1996) 5063.

[8] Müller M., Wittmer J. P. and Cates M. E., Phys.Rev.E, 61 (2000) 4078.

[9] Wittmer J., Paul W. and Binder K., Macromolecules, 25 (1992) 7211.

[10] Carmesin I. and Kremer K., Macromolecules, 21 (1988) 2819.

[11] Paul W., Binder K., Heermann D. and Kremer K., J. Phys. II, 1 (1991) 37.

[12] We describe as 'entangled-like' a system in which the diffusion constant follows a $D \propto N^{-2}$ scaling, to emphasize that such a behaviour is not sufficient to assess the validity of the reptation picture.

[13] FALLER R., preprint cond-mat/0005192.

[14] Fetters L. J. et al., Macromolecules, 32 (1999) 6847.

[15] The so-called "packing length" $p$ is sometimes prefered to the blob size $\xi$ [14. Closer inspection shows that $p$ is essentially the same as $\xi$. Following experiment 14 we have verified the scaling of fig. 4 using $p$ instead of $\xi$. This gives very similar results; the collapse with $\xi \sim d_{e}$ being slightly better.

[16] McKenna G. B. et al., Macromolecules, 20 (1987) 498; Ederlé Y. et al., Materials Science and Technology, A Comprehensive Treatment, edited by A. D. Schlüter, Vol. Synthesis of Polymers (Wiley-VCH, Weinheim-New-York)1999, p. 622.

[17] Obviously, this sets an upper bound $b_{0}(\sigma) \ll \xi(\sigma)$ on the stiffness variation. For very large stiffness there are additional caveats: (i) The structure of the underlying monomer fluid changes with $\sigma$. This effect is partially captured by the stiffness dependence of the mobility $m$, but stiffness and density effects will not decouple for large $\sigma$. (ii) For even larger $\sigma$ the system must undergo a transition to a nematic structure. (iii) For large $\sigma$ and finite $N$ the large scale conformations differ from the properties of flexible molecules, i.e., chains become rods and rings beome circles.

[18] Ebert U. et al., J. Stat. Phys., 90 (1998) 1325; European Phys. J. B, 10 (1999) 105.

[19] This clarifies various recent queries about the bad performance of the Rouse model in the melt limit at high frequencies. Strictly speaking, the Rouse description is only marginally valid in the melt limit $\xi \rightarrow 0$ and no proper Rouse plateau is expected there. This statement is in agreement with fig. 2 and recent careful studies $107,9,11,20$ which clearly show that the forces on short chains in the melt are always correlated, i.e. are not of Langevin type. As soon as the chains overlap this must affect the dynamics because $d_{e} \sim \xi$; the effective forces on reference chains seen are, hence, crossover effects. However, because $d_{e} / \xi \gg 1$ it is allowed to view the Rouse assumption as a useful approximation for various, but not all observed quantities. Note that the experimental practice is to tune the dynamic behaviour of short chains to the Rouse description by means of chain length dependent mobility corrections [3]. This may be misleading when assessing the validity of the Rouse model at small chain length in the melt limit.

[20] Paul W., Smith G. D. and Yoon D. Y., Macromolecules, 30 (1997) 7772. 